\title{
Why We Still Do Not Know What a "Real" Argument Is
}

\section{G.C. GoDdU}

\author{
Department of Philosophy \\ University of Richmond \\ 28 Westhampton Way, University of Richmond, VA 23173 \\ USA \\ ggoddu@richmond.edu
}

\begin{abstract}
In his recent paper, "What a Real Argument is," Ben Hamby attempts to provide an adequate theoretical account of "real" arguments. In this paper I present and evaluate both Hamby's motivation for distinguishing "real" from non-"real" arguments and his articulation of the distinction. I argue that neither is adequate to ground a theoretically significant class of "real" arguments, for the articulation fails to pick out a stable proper subclass of all arguments that is simultaneously both theoretically relevant and a proper subclass of all arguments.
\end{abstract}

Résumé: Dans son récent article, «What a Real Argument is», Ben Hamby tente de fournir une explication théorique adéquate des arguments «réels». Dans cet article, je présente et évalue la motivation de Hamby pour distinguer les arguments «réels» des arguments "non-réels" et pour communiquer cette distinction. Je soutiens qu'aucune de ces motivations est suffisante pour justifier une classe théoriquement importante d'arguments «réels», car il ne réussit pas à identifier une sous-classe appropriée stable de tous les arguments qui est en même temps à la fois théoriquement pertinente et une sous-classe appropriée de tous les arguments.

Keywords: argument, "real", prospective use, Hamby, theoretical relevance

\section{Introduction}

Are "real" arguments a theoretically significant subclass of arguments? In "What is a 'Real' Argument?" I extensively argued "no". However, given the difficulties of conclusively proving a negative, I left open the challenge to advocates of "real" arguments to provide a viable articulation of the notion of "real" argument. In "What a Real Argument Is", Ben Hamby takes up that challenge. He offers both a motivation for the notion of "real" argument and a proposed articulation. 
In what follows, I present and evaluate both the motivation and the articulation. I shall argue that neither is adequate to ground a theoretically significant class of "real" arguments. I begin with the motivation.

\section{The Motivation}

Why bother trying to distinguish, within the class of genuine arguments, so called "real" arguments from non-"real" arguments? Traditional answers have included demarcating the subject matter or scope of informal logic or showing the shortcomings of formal logic. According to Hamby, however, "real" arguments "serve the important theoretical use of demarcating the arguments that should be studied in a basic reasoning course"(Hamby 2012, p. 313). But why think that demarcating the arguments that should be studied in a basic reasoning course is a theoretical use of the distinction? A correct theory of arguments could articulate the ontology and types of arguments even if, for perhaps bizarre sociological reasons, there were no basic reasoning courses. But then, if the theory had a reason for distinguishing "real" and "unreal" arguments, it would not be to identify "the arguments that should be studied in a basic reasoning course."

Granted, if there is a legitimate distinction between "unreal" and "real" arguments, and "real" arguments meet the pedagogical demands of basic reasoning courses, then there would be pedagogical reasons to focus on "real" arguments in such courses. Of course, if "real" arguments did not meet the pedagogical demands of basic reasoning courses, then there would be pedagogical reasons not to focus on such arguments. Either way the legitimacy of the distinction is prior to the determination of the focus of basic reasoning courses. In addition, the determination of focus is ultimately determined by the pedagogical demands and not the theoretical legitimacy of the distinction.

But suppose the distinction is theoretically illegitimate. Would that be enough to delegitimize any pedagogical use of the distinction regardless of pedagogical demands? If it would, then granting a legitimate pedagogical role to "real" arguments means there is some theoretically legitimate underpinning. Indeed, towards the end of his paper, Hamby writes: "if there is a legitimate pedagogical use for the distinction, then we should hope that that distinction pulls some theoretical weight"(Hamby 2012, p. 323).

(C) G.C. Goddu. Informal Logic, Vol. 34, No. 1 (2014), pp. 62-76. 
I flat out deny the conditional. Legitimate pedagogical uses need not be grounded in accurate theory. For example, there is a very pedagogically useful class of frictionless inclined planes in physics classes, but such planes have no theoretical place at all. No adequate physical theory suggests there are such entities. In fact our best physical theories suggest that such entities are impossible. Regardless, getting students to begin initial calculations of accelerations or forces while ignoring friction is pedagogically useful. Pedagogically we may distinguish between high premise arguments and low premise arguments (and pedagogically prefer the latter), but there is no theoretical relevance to typing arguments by the number of premises.

Suppose there is a roughly delineated class of arguments that could be pointed to, for pedagogical reasons, as the proper basis of critical thinking or basic reasoning courses. Should (or must) our theory of arguments include a demarcation of such arguments? Not necessarily.

Firstly, Hamby acknowledges that not all "real" arguments are good candidates for a basic reasoning course. "Such arguments could be considered pedagogically unattractive because they require too much knowledge even to get off the ground"(Hamby 2012, p. 318). I also suspect that there are pedagogical reasons for using allegedly, prospectively unusable arguments to make an educational point-for example, one might use a bizarre example such as, "If Socrates is a chicken, then Socrates has feathers; Socrates has feathers, so Socrates is a chicken", to get students to focus on the form rather than the content. Because the content is bizarre or absurd, students discount it as the relevant feature and focus instead on the pattern of reasoning - exactly what might be desired in an initial presentation of the fallacy of affirming the consequent.

So the class of arguments that should be the focus of a basic reasoning course is certainly smaller than the class of "real" arguments, and some of the arguments presented in such a course may, for pedagogical reasons, be outside the bounds of "real" arguments altogether. Hence, pointing to arguments that should be the focus of a basic reasoning class as the motivation for a theoretical subclass of "real" arguments is inconclusive at best.

Secondly, there are pedagogical reasons for selecting certain arguments as the target of analysis in a basic reasoning class rather than others. Complexity and background knowledge required for understanding are certainly relevant criteria. I strongly suspect that "likelihood of use in contexts of relevance

(C) G.C. Goddu. Informal Logic, Vol. 34, No. 1 (2014), pp. 62-76. 
to our students" could be added as a selection criterion. I suspect that we can, for the most part, easily distinguish those arguments more likely to see use from those less likely to see use. But none of these pedagogical criteria are themselves theoretically relevant. Our theory of arguments need not distinguish complex arguments from non-complex arguments ${ }^{1}$, or distinguish arguments requiring significant background knowledge from those that do not. Arguments about the secession of Quebec may be relevant for Canadian students and so get chosen for inclusion in a Canadian critical thinking class, but not at all relevant for American students and so not get selected for an American critical thinking class. But again, we are not thereby led to believe that there is a theoretically significant distinction between arguments about the secession of Quebec and those not, or between arguments relevant to Canadian students and those relevant to American students. More generally, since the pedagogical criteria themselves do not appear to be theoretically relevant distinctions, we should not think that a class of arguments that satisfies the pedagogical constraints is a theoretically relevant class of arguments.

To sum up, I am not moved by the pedagogical motivation that Hamby offers. While we want what we teach to be ultimately grounded in the truth, we quite legitimately idealize and simplify that truth in order to get our students firmly directed towards it. Hence, pointing at a potentially legitimate pedagogical distinction does not necessarily indicate that a theoretically legitimate distinction lurks underneath. Additionally, we can delineate a rough and ready class of arguments for basic reasoning or critical thinking classes on the basis of criteria such as "not requiring too much background knowledge", "having a certain level of complexity", "having prospective use", or "being relevant to our students", etc., without our theory of arguments demarcating a subclass of arguments that are the "real" ones.

Even though we should not be moved by Hamby's motivation for making the distinction, Hamby may still have provided a distinction that can serve as a theoretically legitimate

\footnotetext{
1 There is a (contentious) theoretical use of 'simple' and 'complex' with regards to arguments that should not be confused with pedagogical judgments concerning argumentative complexity. A 'simple' argument is one with a single conclusion, but some of those arguments can be too convoluted and complex for a basic reasoning course. A 'complex' argument is one composed of multiple simple arguments interconnected in various ways, but many of these are straightforward enough for inclusion in a basic reasoning course.
}

(C) G.C. Goddu. Informal Logic, Vol. 34, No. 1 (2014), pp. 62-76. 
distinction between "real" and "unreal" arguments. I turn to his proffered distinction in the next section.

\section{The Articulation}

According to Hamby "a real argument ... is a practical argument, in the sense that it serves or could serve as a tool of persuasion or for some other use in the context of the communicative practice of establishing good candidates for belief and action"(Hamby 2012, p. 321). He also describes "real" arguments as "prospectively useful" arguments where "an argument has prospective usefulness when it could be offered in practice to support a controversial candidate claim that calls for judgment in the context of deciding what to believe or do"( $p$. 313). Hamby claims these sorts of arguments "matter to people substantively"(p. 313), "are relevant arguments with conclusions that matter to people in substantive ways" (p. 314), and are "non-trivial arguments that matter in real-life"(p. 314). These arguments are to be contrasted with the arguments "that are not used, nor could prospectively be used, to some end of argumentation in the practice of forming beliefs and deciding what to do"(p. 324).

"Real" arguments then are substantial, relevant, nontrivial, matter in real life, and involve controversial claims. Since these descriptors are not synonyms there is already a problem of demarcation. Is satisfying all the descriptors a requirement for an argument to be "real" or must an argument merely satisfy at least one? Even if we suppose that an argument that satisfies none of the descriptors is definitely not a "real" argument, and one that satisfies all of them definitely is "real", we still will not know what to do with mixed cases. In an uncontested theocracy, the conclusion 'God exists' will be uncontroversial, yet still substantial. Whether Mozambique will ever invade Zimbabwe may be substantial, yet irrelevant to our concerns. Whether objects can be dispensed with in favor of properties in a logical model may be controversial, but will not matter in real life. Are the arguments in these cases "real" or not? Given that we want our theoretical categories to be exhaustive, for any argument we should, in principle, be able to tell whether it is "real" or not-so far this is not true of Hamby's articulations.

Suppose, however, that the status of the mixed cases is clarified. Regardless, whether a conclusion or argument is substantial, controversial, relevant, or matters in real life 
depends upon our interests and goals. Whether concrete physical objects are metaphysically real is controversial and matters to (some) philosophers, but is irrelevant to most everyone else. Whether two angels can occupy the same space was a substantial issue for Aquinas, but was inane from the perspective of critics of scholasticism. Arguing for the conclusion that "the whole is greater than its parts" would have been considered an exercise in triviality for centuries, but post Cantor and Dedekind arguing for it would be controversial. What is substantial, relevant, or controversial to one person may not be so to another. But if, say, "being substantial" is a criterion of "real" arguments, then an argument can be "real" for one person and not "real" for another. Our theory of arguments should eschew such relativistic categories. We do not want our theoretical ontology to be determined by our interests and goals; we are supposed to discover and articulate the way the world is, not the way we want it to be.

One option for the advocates of "real" arguments is to claim that there is an objective category of "substantial" arguments or "relevant" arguments. Aquinas was either giving a substantial argument concerning angels occupying the same space or he was not, and if he was not then he was not giving a "real" argument. (I admit that what the objective basis for an argument "being substantial" might be is beyond me.) Another option is to claim that arguments are "real" if they are substantial or relevant or controversial for even one individual. Unfortunately for the advocates of "real" arguments, both options run into difficulties once we add in the "actually used" or "prospectively used" aspects of Hamby's proposed distinction. I begin with "actually used".

Does an argument that is actually used in the practice of forming beliefs and deciding what to do (or persuading others in this regard) automatically count as a real argument? On the one hand Hamby should say "yes" since non-"real" arguments are, according to him, those that are not used nor could prospectively be used to some end of argumentation. Since they actually are used, the arguments count as "real". But on the other hand "real" arguments are supposed to support controversial candidate claims, or be non-trivial arguments that matter in real life, or have conclusions that matter to people in substantive ways. In the latter case, genuine arguments that have actually been used to argue for uncontroversial claims or that do not matter to people (despite what the proponents of the argument might think) are not "real". Hence, if we respect both the "unreal could not be used" and the "real are substantial, controversial, etc."

(C) G.C. Goddu. Informal Logic, Vol. 34, No. 1 (2014), pp. 62-76. 
aspects of Hamby's demarcation, then actually used trivial arguments will count as both "real" and not "real", which is a theoretical non-starter. But if we can only respect at most one aspect, which should it be?

Suppose that there is an objective fact of the matter, independent of our attitudes, whether a given argument is substantive (or controversial or relevant) or not. Suppose further that at least some of the criticisms charging actual arguments used in argumentative contexts with being trivial are correct. If actual use in an argumentative context is sufficient for an argument to count as "real", then Hamby needs to drop the descriptors "relevant", "substantial", "controversial", etc., since arguments that fail to meet these criteria have actually been used in practice (and have been criticized for failing to meet these criteria). For example, at least part of Donald Davidson's argument (Davidson, 1973) against conceptual schemes is that, understood in a particular way, the claim the conceptual relativists are arguing for is trivial or uncontroversial. But if Davidson is right that the claim being argued for is trivial or uncontroversial, then we have actual arguments (and so "real" arguments) concerning what is trivial or uncontroversial. More generally, to deny that any actual arguments have been made for what is trivial or uncontroversial - to claim that "preaching to the choir" has never occurred in argumentative contexts-seems problematic at best.

On the other hand, if actual "real" arguments must not only be actually used, but also be substantive or relevant or concern what is controversial, then, assuming Davidson is right, the conceptual relativists were not giving "real" arguments; nor, if the critics of Aquinas are right, was Aquinas giving a "real" argument for the possibility of two angels occupying the same space. But charging arguers with arguing for something already accepted or uninteresting has a long history. If, however, these arguments are not "real", and the focus of basic reasoning courses is supposed to be "real" arguments, then teaching these arguments (and the general "show arguing for something uninteresting" strategy) is also not proper in a basic reasoning class. That seems wrong. If these arguments are reasonable targets of instruction and criticism despite not being "real", then once again Hamby's motivation for making the distinction is not lining up with the proffered distinction, and I have no idea why we are trying to distinguish "real" arguments from non-"real" ones.

Suppose instead that an argument being substantive or controversial or relevant for at least one individual is sufficient

(C) G.C. Goddu. Informal Logic, Vol. 34, No. 1 (2014), pp. 62-76. 
to make the argument "real". Presuming that the conceptual relativist or Aquinas took their arguments seriously, their arguments would count as "real". More generally, we must separate out our collective judgments of arguments being substantive from what proponents (or receivers) of the arguments might believe. An arguer (or a receiver) believing that an argument has substance or argues for a controversial claim is, on the current supposition, enough to make an argument "real". But one might wonder whether any argument will be excluded on such a liberal understanding of substantial or controversial, etc. Assuming that all actually used arguments have at least one individual who takes them seriously, then all actually used arguments will count as "real", regardless of how insubstantial or trivial they might seem to us. But given that we are not merely interested in arguments that actually have been made, but the ones that could be made, I suspect the exclusion problem will only get worse. I turn next to arguments that "could be made in the context of judging what to do or believe".

Recall that according to Hamby, "real" arguments are ones that "could be offered in practice to support a controversial candidate claim that calls for judgment in the context of deciding what to believe or do"(Hamby 2012, p. 313), or "could serve as a tool of persuasion or for some other use in the context of the communicative practice of establishing good candidates for belief and action"(p. 321); whereas non-"real" arguments are "instances of genuine arguments that are not used, nor could prospectively be used, to some end of argumentation in the practice of forming beliefs and deciding what to do"(p. 324). Hamby argues that there are genuine arguments on both sides of the "could be used/could not be used" divide. I shall argue that interpreting the divide so that there are arguments on both sides will make the divide theoretically irrelevant. Attempting to avoid the charge of irrelevance will require making the "could not be used" side of the divide empty. But if one side of the divide is empty, then the divide is still theoretically irrelevant. Hence, either way, the divide is theoretically irrelevant.

Consider an argument concerning whether the sun will generate an Earth threatening solar flare in the next 50 years. Clearly such a conclusion matters to us and is significant to what we do and believe. But also suppose that this argument, given the complexity of the interior of the sun, has a million premises. Though there is a perfectly coherent sense in which it is not practical for us, limited as we are, to use this argument, it is certainly an argument that concerns something that matters, is substantive, and so satisfies most of the descriptors Hamby uses

(C) G.C. Goddu. Informal Logic, Vol. 34, No. 1 (2014), pp. 62-76. 
to clarify what a "real" argument is. So is this hypothetical argument a "real" argument or not?

If it is not, because of the lack of "prospective use", then once again Hamby's descriptors do not capture "real" arguments. But more importantly, if it is not a "real" argument, then the range of "real" arguments seems quite parochial, limited by our current abilities and knowledge. Since Cicero could not use any argument in quantum physics, does it follow that they were not "real" then, but are "real" now? The arguments that eight-year-olds can use are different than those that many grown adults can practically use-so are the arguments we can use, but they cannot, "real" for us and not "real" for them and vice versa? Again, this sort of relativism is to be avoided in our theoretical ontology.

If the hypothetical solar flare argument is a "real" argument (or the eight-year-old's simplistic arguments are "real"), then we must understand "could be used" quite liberally. If even one potential arguer could use the argument in the context of deciding what to do or believe, then the argument will count as "real". (If we hold that an argument could be used if at least one arguer [or receiver] holds the argument to be significant or relevant or controversial, then this option lines up with the option we considered previously.) But if just one potential user is sufficient to make an argument "real", then it is hard to see what arguments might be excluded by Hamby's account. Arguments that seem inane to us may seem perfectly substantial and reasonable to use for those less intellectually perspicuous than we are, just as many of our substantial arguments may seem inane to Laplacian super-geniuses. Also if an omnipotent being counts as a potential arguer and an omnipotent being can do anything logically possible, then such a being could use any argument just so long as it was not logically impossible to use such an argument. But are there any genuine arguments that are logically impossible to use? Using them in the context of deciding what to do or believe would entail a contradiction?

In general the problem is as follows: in its most general sense "could be used in the context of judging what to do or believe" does not exclude any arguments (or if it does, I do not yet know what these arguments are) in which case "real" is just another word for genuine argument and Hamby has already conceded that "real" arguments are supposed to be a proper subclass of genuine arguments (Hamby 2012, p. 324). Understanding "could be used" as "could be used by us (or those intellectually like us) in normal circumstances" introduces a

(C) G.C. Goddu. Informal Logic, Vol. 34, No. 1 (2014), pp. 62-76. 
parochialism and relativism into our categories that have no place in our theoretical ontology. Take all the arguments that ever were, are, or will be and all the genuine arguments that ever could have been or could be. Is there a genuine stable proper subset of those arguments that are the "real" ones? That is the generality we seek in our theoretical ontology.

I doubt that there is a stable middle ground. At the same time, I admit that the examples in the previous paragraphs, while suggestive, do not conclusively prove that there is no stable middle ground for "could be used". Regardless, if there were a substantial theoretical payoff (or even any theoretical payoff) to the notion of a "real" argument, doubts about a theoretically stable use of "could be used" would not be enough to warrant giving up the chase. But the advocates of "real" arguments have yet to provide a reason to demarcate "real" arguments that comes close to corresponding to what they think "real" arguments might be.

Suppose for the moment, however, that we take up the challenge of trying to find a sense of 'could be used' that charts a stable middle ground between allowing in all genuine arguments and being so parochial or relative in scope that it has no theoretical merit. Whatever this alleged middle ground is, Hamby has yet to find it. Consider his example:

Socrates was a man; All men are mortal; therefore, Socrates is mortal.

According to Hamby, "this argument is not real, because it is unlikely that anyone would ever use it to support its uncontroversial conclusion"(Hamby 2012, p. 321). But "real" arguments are arguments that could be used as tools of persuasion (amongst other possible uses in argumentative contexts). Even if it is unlikely that the Socrates argument be so used, it still presumably could be so used and so fits Hamby's articulation of "real" argument. Consider Plato's arguments about the immortality of the soul and that Socrates is better identified with that soul than his body. Now imagine a contemporary of Plato trying to rebut Plato's arguments by appeal to common sense. He argues by reminding his audience that all men are mortal and that Socrates was a man, in which case they must conclude that Socrates is mortal. The audience agrees and the contemporary goes on to use the conclusion that Socrates is mortal to argue that Plato's view about Socrates' immortality, or that Socrates is better identified with his soul 
rather than his body (or the combination of the two) must be wrong. ${ }^{2}$

Plato of course will not be persuaded by this argument, for he will disagree with the "all men are mortal" premise. But even someone who has heard Plato's arguments may judge "all men are mortal" to be more likely true than false and so accept the "Socrates is mortal" conclusion, contra Plato, on the basis of the other two premises. Alternatively, someone might independently believe all men are mortal, but require convincing that Socrates is a man rather than the son of Zeus and a naiad. Upon being convinced of the man-ness of Socrates one could be persuaded, on the basis of the two premises, that Socrates was mortal. If even one of these potential scenarios is one in which the Socrates argument is used in the context of deciding what to do or believe, then the argument is, contra Hamby, a "real" argument.

What about this even more implausible argument:

\section{Lemons are red, so the moon is made of blue cheese.}

Imagine you and I are trapped, or at least our online avatars are trapped, in one of many possible online artificial worlds. Which world we are trapped in matters, since the location of the emergency escape override varies from world to world. I am trying to convince you that we are in the "moon is made of blue cheese" world. Since we both know that all the "lemons are red" worlds are also "moon made of blue cheese" worlds, I can point to the red lemon in my avatar's hand and type "lemons are red, so the moon is made of blue cheese" as a way to convince you we are indeed in a blue cheese moon world, so we should head to location $z .{ }^{3}$ But if the Lemons argument could reasonably be used and so counts as "real", then what arguments will not count as "real"?

\footnotetext{
2 Or imagine trying to console a kindergartner, whose grandfather has just died, with, "All people die eventually, dear." Sad kindergartner: "But Grandma is a person too." "Yes, honey." New round of tears as kindergartner comes to the conclusion that grandma will eventually die.

3 Note a consequence of this example: trying to find an absolute, objective sense of "relevant" is misguided-anything can be made relevant to anything else. A more interesting question is: given a certain context of fixed facts, is $a$ relevant to $b$ ? See also David Botting, 2013.
}

(C) G.C. Goddu. Informal Logic, Vol. 34, No. 1 (2014), pp. 62-76. 


\section{Mere Examples}

I am skeptical that there are any arguments that fail to be such that they "could be used, or even reasonably used, in the context of deciding what to do or to believe." ${ }^{4}$ What follows is a very general argument for that claim-arguing against it is selfdefeating.

I take the existence or non-existence of "real" arguments to be a substantial point of contention between us. It matters for what we ought to believe, given we are interested in the truth. Suppose Hamby insists that the examples I gave above are not "real" arguments. Or suppose that Hamby grants my claims about the above examples, but offers yet other examples. To convince me that his examples are not "real" arguments, Hamby has to argue that the proposed examples fail to be "real" arguments. I presume such an argument will go like this: $\mathrm{X}$ lacks the properties required for an argument to be a "real" argument; hence, $\mathrm{X}$ is not a "real" argument. But how can Hamby, or anyone, make that argument without using $\mathrm{X}$ within the context of deciding what to do or believe?

Claiming that $\mathrm{X}$ is merely part of the main argument will not do. $\mathrm{X}$ is an argument and it is "used in argumentative practice to form beliefs and make decisions" and so satisfies Hamby's account. But, defenders of "real" arguments might

\footnotetext{
4 At one point in his paper, Hamby takes me to task for quickly dismissing a restriction on use like "reasonable" use (Hamby 2012, p. 323). I dismissed it only insofar as no articulation of what could be meant by "reasonably used" had been offered, so no theoretically relevant or significant definition of "real" argument in terms of "reasonable use" has been provided. Hamby still has not provided an articulation of "reasonable use" in his current paper. Surely it is up to the defenders of "real" argument to provide such an articulation.

I admit that I was, and remain, skeptical that a theoretically adequate articulation could be provided. Some of my reasons have been articulated here: reasonableness, like consequentialness or relevance, is dependent on our goals and interests such that an argument might be reasonably used by one person, but not another. Also, the use of some arguments in a particular context might be criticized precisely because the use of them in that context is unreasonable. But if people actually use such arguments and it is worth teaching students this sort of criticism, then they seem a prime candidate for basic reasoning courses; and the distinction, and the alleged motivation for the distinction do not mesh. Finally, I am deeply suspicious of trying to build reasonableness in at the level of our theoretical ontology. Reasonableness, in conjunction with our variable goals and interests, is supposed to be a consequence of our theory, not a primitive within it.
} 
respond, $\mathrm{X}$ is merely being mentioned or $\mathrm{X}$ is being used as a mere example or $X$ is not being used to argue for $X$ 's conclusion. "Real" arguments, in contrast, can be used to argue for their conclusions. As Hamby claims at one point, "real" arguments are "not constructed merely for the sake of illustration, with no substantive content"(Hamby 2012, p. 322). So "real" arguments are not just arguments that could be used in the context of deciding what to do or believe; rather they are arguments that could be used to argue for their own conclusions and not merely used as examples or mentioned within the context of deciding what to do or believe.

Firstly, I doubt there is a clean distinction between using an argument to argue for its conclusion and using an argument as an example. Here is an example from Roy Sorensen (1996):

Some arguments are composed solely of existential generalizations, so some arguments are composed solely of existential generalizations.

The argument is used to argue for its conclusion on the very basis that it is an example of that sort of argument. Or consider:

Petunias prance proudly past the pool, so some "unreal" arguments have absurd premises.

Suppose this is not a "real" argument. But then it is an instance of an "unreal" argument with an absurd premise. Since the argument exemplifies the conclusion ${ }^{5}$, it should convince me of the truth of its conclusion, in which case we have an "unreal" argument that can be used to argue for the truth of its conclusion. Hence, according to our modified definition of "real" argument and contra our initial supposition, it is a "real" argument.

Secondly, adding the restriction that "unreal" arguments are ones that can never be used to argue, except as examples, does not sidestep the issues raised in the previous section. If the Lemons argument can be reasonably used, in at least some contexts, to argue for its conclusion, then I am hard pressed to see how to construct an argument that could not be used to argue for its conclusion in any context. What properties would such an argument have? Totally (seemingly) irrelevant premises would not be enough; nor would obviously false premises or an obviously true conclusion, since what is obvious to us may not

\footnotetext{
${ }^{5}$ See Goddu 2012 for more discussion on exemplification and argument.
}

(C) G.C. Goddu. Informal Logic, Vol. 34, No. 1 (2014), pp. 62-76. 
be obvious to others. In other counterfactual situations those premises could be true and in yet others that conclusion false. Unless we are going to rule dialethism (or the theological view that an omnipotent being can do anything, even bring about contradictions) out of court by fiat, we cannot even rule out the possibility that someone might argue using contradictory premises or argue for a contradictory conclusion. Once again we are in the position in which we either make parochial restrictions on what "can be used to argue for its conclusion" means, in which case the distinction will not be part of our correct theory of argument, or no genuine argument will be one that could only ever be used as an example or merely mentioned, in which case there is no distinction to be made. Either way there is no theoretically significant subclass of the class of genuine arguments that is the class of "real" arguments.

\section{Conclusion}

One should not think that I am denying that there are substantive arguments concerning controversial matters, or that I do not think that we are quite good at distinguishing substantive from trivial arguments, or useful arguments from non-useful arguments, or arguments that are more likely to see use from those that are not. I suspect we are quite good at making these discriminations and certainly good enough to make them such that if a certain subgroup of such arguments meets other pedagogical criteria, we could easily use such arguments as the basis of basic reasoning courses, at least as taught in the early $21^{\text {st }}$ century. But all of this can be explained by appeal to a general, though certainly not universal, congruence of our abilities, interests, and background knowledge and not by an appeal to some underlying subclass of "real" arguments.

What I do deny is that we have any good reason to think that our correct theory of arguments will have a distinction between "real" or "practical" and non-"real" or "impractical" arguments within it. Even if it turns out that there is a subclass of "real" arguments, I still have no idea what this class is and no reason to think such a subclass is theoretically relevant.

\section{Acknowledgements}

Thanks go to two anonymous referees of Informal Logic for helpful comments. Additional thanks to Brannon McDaniel and 
Michele Bedsaul for comments and proofing of earlier drafts. An earlier version of this paper was presented at OSSA 2013, Virtues of Argumentation. I am grateful to the audience and my commentator, David Botting, for stimulating questions.

\section{References}

Botting, D. (2013). "The Irrelevance of Relevance." Informal Logic 33, 1-21.

Davidson, D. (1973). "On the Very Idea of a Conceptual Scheme." Proceedings and Addresses of the American Philosophical Association 47, 5-20.

Goddu, G.C. (2012). "Exemplification and Argument." Canadian Journal of Philosophy 42, 235-254.

Goddu, G.C. (2009). "What Is a 'Real' Argument?" Informal Logic 29, 1-14.

Hamby, B. (2012). "What a Real Argument Is." Informal Logic $32,312-325$.

Sorensen, R. (1996). “'P, Therefore P' Without Circularity." Journal of Philosophy 88, 245-266. 